\title{
DEEP: Density-Aware Emergency Message Extension Protocol for VANETs
}

\author{
Ming-Chin Chuang and Meng Chang Chen
}

\begin{abstract}
With the rapid developments in vehicular communication technology, academics and industry researchers are paying increasing attention to vehicular ad hoc networks (VANETs). In VANETs, dissemination delay and reliability are important criteria for many applications, especially for emergency messages. Existing approaches have difficulty satisfying both requirements simultaneously because they conflict with one another. In this paper, we propose a novel mechanism, called the Density-aware Emergency message Extension Protocol (DEEP) to disseminate emergency messages in VANETs. DEEP resolves the broadcast storm problem, achieves low dissemination delay, and provides high reliability over a realistic multi-lane freeway scenario. The mechanism delivers emergency messages to a specific area (e.g., the area before the exit) in a timely manner and guarantees that all relevant vehicles in that area will receive the messages. Drivers can then change their routes and avoid getting caught in a traffic jam. Performance evaluations via NS-2 simulations demonstrate that DEEP achieves both lower dissemination delay and higher reliability than existing approaches.
\end{abstract}

Index Terms-VANETs, emergency message dissemination, reliability, multi-lane freeway.

\section{INTRODUCTION}

$\mathbf{V}$ EHICULAR Ad Hoc Networks (VANETs) are becoming increasingly popular. Because of advances in technology, such as the cheaper Global Position Systems (GPS), powersaving embedded computers, and the proliferation of Internet and web applications, people can enjoy many convenient services while they are driving. Moreover, the VANET nodes have abundant energy, storage, and computational capability.

In a VANET, the network topology changes rapidly due to the high mobility of vehicles (i.e., ad hoc nodes), but the vehicle motion is constrained by the road topology and layout resulting in the movement of the vehicles being predictable. Based on the characteristics of wireless devices, communication modes are divided into two types: Vehicle-to-Vehicle (Vto-V) and Vehicle-to-Infrastructure (V-to-I) modes, as shown in Fig. 1. The technology is called Dedicated Short Range Communications (DSRC) [1]. For the communication protocol, the U.S. Federal Communications Commission (FCC) assigned the frequency spectrum of $75 \mathrm{MHz}$ to VANETs. The most common messages transmitted in VANETs are safety and infotainment messages. Safety messages warn drivers about potential hazards, such as accidents, and provide up

Manuscript received November 2, 2012; revised June 10, 2013; accepted July 30,2013 . The associate editor coordinating the review of this paper and approving it for publication was $\mathrm{H}$. Hassanein.

M.-C. Chuang is with the Research Center for Information Technology Innovation, Academia Sinica, Taiwan (e-mail: speedboy@gmail.com).

M.-C. Chen is with the Institute of Information Science, Academia Sinica, Taiwan (e-mail: mcc@iis.sinica.edu.tw).

Digital Object Identifier 10.1109/TWC.2013.090413.121697

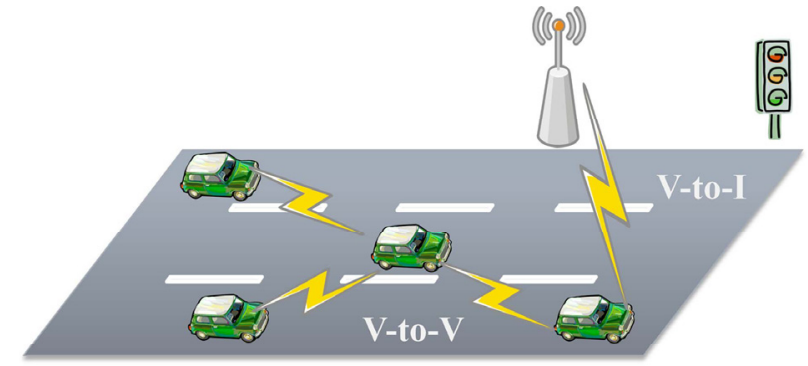

Fig. 1. Two types of communication modes in VANETs.

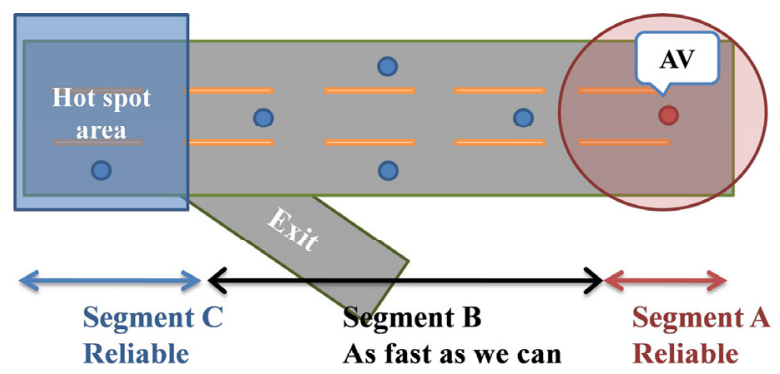

Fig. 2. The operations of the emergency message dissemination mechanism on a freeway.

to date information (e.g., road condition warning, the use of emergency electronic brake lights, and safe distance sensing) in front of the vehicle, even if the line of sight is bad. Drivers can change lanes or take other action to adjust to the abnormal conditions [2]. For example, if there is sufficient time, drivers may be able to take an alternative route and avoid getting stuck in a traffic jam. Infotainment messages are used for other applications, such as providing information about shopping, parking lots and the weather. In this paper, we focus on the dissemination of the safety/emergency messages in VANETs.

Yang et al. [3] observed that about $60 \%$ of road accidents could be avoided if drivers were given a warning at least a half-second prior to a collision. This calls for an efficient emergency message (safety message) dissemination mechanism for VANETs. Moreover, the efficient emergency message dissemination is able to assist the real-time decision making for autonomous vehicle [4]. Therefore, two of the most important requirements of emergency message dissemination are low delay and high reliability. However, it is difficult to satisfy the requirements simultaneously because they conflict with one another. Emergency messages must be broadcasted quickly and disseminated efficiently [5], but this usually results in the broadcast storm problem [6] and unreliable transmissions. 
In this paper, we propose a novel mechanism, called the Density-aware Emergency message Extension Protocol (DEEP), to disseminate emergency messages in VANETs. Figure 2 shows the operations of the proposed emergency message dissemination mechanism over a realistic multi-lane freeway scenario when there is an accident. The freeway is divided into three segments in our scenario when an accident happens. We define the hot spot area (i.e., segment $\mathrm{C}$ in the figure) as the rectangular area before the exit. The exit is a branch of the freeway and it can split the traffic for avoiding the traffic jams. The mechanism's most important function is to ensure that all vehicles in the hot spot area receive emergency messages as soon as possible. Thus, drivers can change their routes to the exit and avoid the heavy traffic jam caused by the accident. In addition, all the vehicles in segment A of the figure should receive the message so that they can avoid the accident caused by the abnormal vehicle (AV). In this paper, "reliable" means that all relevant vehicles can receive the emergency messages. Consequently, the dissemination of the messages in segments $\mathrm{A}$ and $\mathrm{C}$ must be reliable. On the contrary, in segment $\mathrm{B}$, the messages should be forwarded as quickly as possible to reduce the delay to the hot spot area. We focus on the freeway scenario because if the vehicles miss the nearest exit to the AV, they may waste a great deal of time caught in a heavy traffic jam. Most existing approaches [3] [7] broadcast messages reliably in segment A, but they do not consider the forwarding and reliability issues in segments $\mathrm{B}$ and $\mathrm{C}$, so vehicles may still get caught in a traffic jam. Therefore, in this paper, we focus on the fast forwarding and reliability issues in segments $\mathrm{B}$ and $\mathrm{C}$ respectively. Note that, although the proposed fast forwarding mechanism for segment B does not guarantee that all vehicles in the segment will receive an emergency message, it guarantees that the message will be forwarded to segment $C$. This is because the mechanism ensures that the message is forwarded to next vehicle successfully.

The remainder of this paper is organized as follows. Section II contains a review of related works. In Section III, we describe the proposed DEEP; and in Section IV, we present the simulation results based on ns-2. We then summarize our conclusions in Section V.

\section{RELATED WORK}

Mohapatra et al. [5] and Chen [8] provide a summary of message broadcast schemes in VANETs. Such schemes have three objectives: (1) reduce the number of redundant messages; (2) guarantee fast forwarding of emergency messages; and (3) ensure dissemination of the messages is highly reliable.

For restraining the communication overhead, a number of studies [9-12] use a clustering scheme to mitigate the redundant message problem. Most of the existing VANET clustering algorithms are derived from the MANET clustering schemes [26-28], and the studies [29] [30] show the benefits of clusters including the reduction of communication overhead and improvement of delivery ratio. Unfortunately, the creation and maintenance of the cluster structure in a highly mobile network is impractical, because of the continuous variation of the network topology [31]. As a result, the cluster membership changes frequently and then leads to extra delay and computation overhead for reconstructing the cluster. Tseng et al. [6] proposed counter-based, location-based, and adaptive hello message schemes to mitigate the broadcast storm problems in mobile ad hoc networks. However, these schemes cannot satisfy the requirements of fast forwarding and reliability.

In the fast forwarding aspect, the random time slot method is straightforward; however, it only reduces the collision probability but not guarantee fast forwarding. The node with smallest random number may not be the farthest node from the source node since the random time slot method does not consider the distance between the source node and the receive node. Fasolo et al. [14] proposed the Smart Broadcast (SB) protocol, which partitions the surface of the transmission range into adjacent sectors and assigns a unique waiting time to each sector. Consequently, nodes located in different sectors have different waiting times. By assigning the minimum waiting time to the sector farthest from the source, SB guarantees that the farthest node will relay the message first and that the message can propagate the longest distance in each hop of the transmission. A similar concept with a different formula for partitioning sectors, called slotted 1-persistence broadcasting, is proposed in [15]. Although using adjacent sectors is a good way to select the relay node, it is difficult to determine the optimal sector size for each scenario. Larger sectors result in shorter forwarding delays due to the smaller number of sectors overall; however, the collision probability increases since vehicles in the same sector have the same waiting time. The methods proposed in [16] [17] assign each node's waiting time based on predefined equations. Specifically, the node farthest away from the source node is assigned the shortest waiting time for fast forwarding. However, under this scheme, it is difficult to define the optimal range of the waiting time for different vehicle densities. Davide et al. [18] proposed a propagation function that yields a shorter waiting time and higher transmission probability if a node is close to the destination. This function ensures that the message is forwarded to the destination correctly. However, as high mobility is the one of characteristics of VANETs, the pre-defined propagation function may not be suitable in complicated real-world scenarios. Chen et al. [25] proposed a location-based back-off scheme to reduce the total number of rebroadcasts, the packet collision rate and the average packet delay. However, this scheme still needs the additional control message to maintain the warning group. Moreover, the vehicle only rebroadcasts once resulting in decreasing of the reliability when it cannot overhear any rebroadcasting message. Consequently, the above fast forwarding schemes do not consider the vehicle density, resulting in high collision probability if the range of the random number is small when the vehicle density is high.

In the reliability aspect, the approach proposed in [11] [19] [20], verifies that a message has been delivered to each receiver correctly because each receiver must send an ACK frame back to the sender. However, the sender needs to maintain a receiver list; otherwise, a message would have to be transmitted via multiple unicast transmissions, resulting in inefficient message dissemination in VANETs. In addition, the ACK mechanism could waste more wireless bandwidth 
and suffer from ACK collision problem. A repetition scheme can also ensure the reliability of a system. For example, the approach proposed in [3] [21] sends the same message several times, so there is a high probability that all relevant users will receive the message successfully. More recently, Wang and Hassan [22] use the blind XOR (BXOR) scheme to reduce the retransmission times (i.e., By XORing multiple packets into a single retransmission, BXOR recovers an increased number of lost packets per retransmission). Although these approaches use a rate decreasing algorithm to reduce the number of redundant messages and the retransmission delay, it does not determine optimal sending rates for different vehicle densities and channel error rates.

\section{Density-AWARE EMERgenCy MESSAGe EXTENSION PROTOCOL (DEEP)}

In this section, we describe the proposed Density-aware Emergency message Extension Protocol (DEEP), which includes the operations of vehicles, the fast forwarding mechanism and the reliability mechanism. DEEP, which focuses on the fast forwarding and reliability issues in segments B and $\mathrm{C}$ respectively, works based on the vehicular density (i.e., it determines the block size and the number of times for retransmission the emergency message based on the vehicular density on the segment of the source vehicle) for low dissemination delay and high reliability. In this paper, the dissemination delay means the delay time when a message is transmitted from the abnormal vehicle to the hot spot area. We assume each vehicle is equipped with a GPS to obtain its geographical location and at least one wireless transceiver. Each vehicle periodically sends its location and speed to the intelligent transportation system (ITS). In this paper, ITS system acts like a database manager. It is responsible for recording the traffic information and forwards the information such as the vehicle density and average vehicle speed to other vehicles in the same segment. Therefore, each vehicle can obtain the information of the car density from ITS through the navigation (i.e., GPS channel), vehicular sensor equipment (i.e., V2V communications), or the RSU's broadcast message (i.e., V2I communications).

\section{A. The operations of vehicles}

DEEP is designed to focus on the fast forwarding and reliability issues in segments $\mathrm{B}$ and $\mathrm{C}$ respectively. In the freeway scenario with multiple lanes, a vehicle is un-flagged initially (i.e., state flag $=0$ ). However, the vehicle becomes a flagged vehicle (i.e., state flag=1) when it enters the segments A and C. The Algorithm I, source vehicle adaptive algorithm (SVAA), can be described by the pseudocode in Figure 3. When the accident happens, the AV broadcasts an emergency message with the vehicle's ID to alert the vehicles behind it. It rebroadcasts the message periodically based on the reliability scheme (described later) to ensure that all vehicles in segment A receive the warning about the accident. Each vehicle that receives the message checks two points: (1) if the message is new (i.e., the message contains a new ID), and (2) if the message was broadcasted by a vehicle further ahead on the freeway. If both points are confirmed, the vehicle forwards the message based on the proposed fast forwarding protocol. The receiving vehicles first check the message's ID to avoid forwarding a redundant emergency message again. Then, based on the location information obtained from the GPS, the vehicles determine their deferral times using DEEP. The vehicle with the shortest deferral time broadcasts the message when its deferral time expires, and then becomes the relay of this hop transmission. If the relay does not hear an emergency message being broadcasted by any vehicle in the timeout period, the forwarding procedure is deemed a failure due to packet collisions, transmission errors or network failure. Then, the collision recovery mechanism of the fast forwarding mechanism is triggered to ensure that the message is delivered to the hot spot area and the block flag of the message is set to 1 . The Algorithm II, following vehicle adaptive algorithm (FVAA), can be described by the pseudocode in Figure 4. When the emergency message is propagated to the hot spot area (i.e., segment $\mathrm{C}$ in Fig. 2), our protocol's reliability scheme is triggered. The last relay vehicle in the hot spot area will continue re-broadcasting the message until another vehicle receives it and takes over as the relay vehicle because it will stay in the area longer. Finally, every vehicle in the hot spot area receives the message, changes the route to its destination, and leaves the freeway to avoid the traffic jam. The source vehicle broadcasts the warning cancel message to the hot spot area via DEEP scheme if the car accident is settled. Then, the following cars will drive on the freeway.

\section{B. Fast Forwarding Mechanism}

In this work, the methods for segmenting blocks include a regular segmentation method and an irregular segmentation method. The former adjusts the length and width of the unit block size simultaneously, while the latter adjusts the length and width arbitrarily.

DEEP is designed for the freeways that the road network topology is linear. We assume that (1) the coverage area of the source can be partitioned in adjacent blocks, as shown in Fig. 5 ; and (2) each vehicle can obtain its location via a GPS and thus knows the block that it belongs to. If the vehicle farthest from the source can forward the message with the shortest waiting time, the forwarding delay in segment $\mathrm{B}$ will be the shortest. Therefore, we assign a deferral time to each block, as shown in Fig. 5. Note that the total number of blocks in the transmission range of the source vehicle is not fixed, but it depends on the size of the block. We discuss this aspect later.

The source vehicle broadcasts the emergency message with a flag in the header to notify the receiving vehicles about the block size in the current relay. Thus, in each round of forwarding, all vehicles in the transmission area have the same block size. In our scheme, when a vehicle receives a new emergency message, it waits for the inter-frame space (IFS) and it calculates and waits for the deferral time based on the distance between the source vehicle rather than waiting a random back-off time. The deferral time is computed as follows:

$T_{\text {defer }}=T_{\text {slot }}\left(\left\lceil\frac{D_{y}^{\max }}{O_{y}}\right\rceil\left(\left\lceil\frac{D_{x}^{\max }}{O_{x}}\right\rceil-\left\lceil\frac{D \cdot \cos \gamma}{O_{x}}\right\rceil\right)+\left\lfloor\frac{D \cdot \sin \gamma}{O_{y}}\right\rfloor\right),(1)$ 


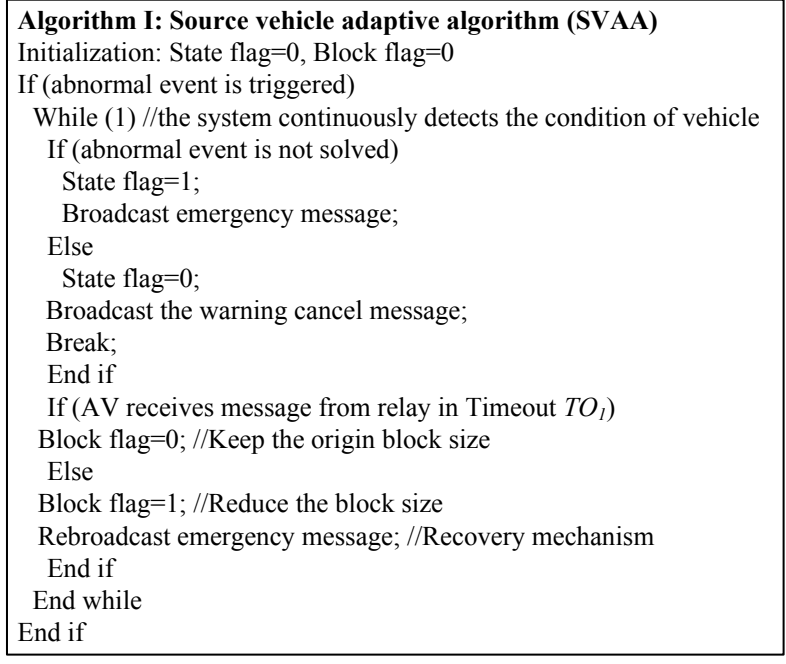

Fig. 3. The operations of the abnormal vehicle (AV).

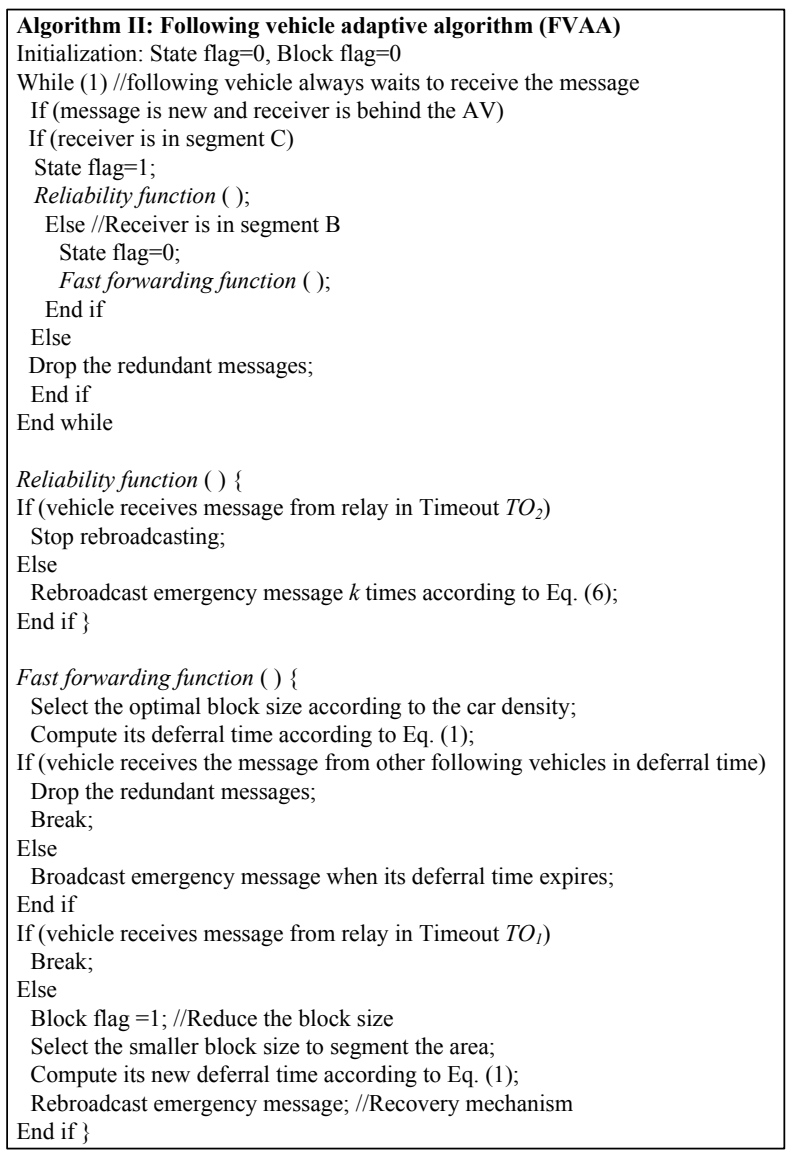

Fig. 4. The operations of a vehicle behind the AV.

where $T_{\text {defer }}$ is the vehicle's deferral time; $T_{\text {slot }}$ is a basic unit of slot time; $O_{x}$, and $O_{y}$ are the length and width of the block size announced by the source vehicle respectively; $D$ and $\gamma$ are the distance and angle between vehicle and the source respectively; and $D_{x}^{\max }$ and $D_{y}^{\max }$ are the transmission ranges of the $\mathrm{X}$-axis and $\mathrm{Y}$-axis respectively. Then, the vehicle with the minimum deferral time will forward the message. However, if the relay vehicle does not hear another forwarding

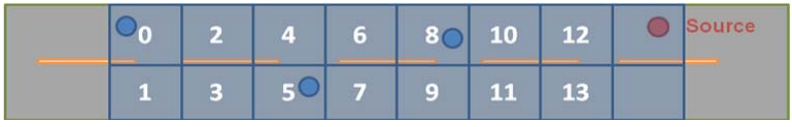

Fig. 5. The setting of deferral times for each block under different distance from the source.

message within the timeout period, the forwarding procedure is deemed a failure due to a collision, transmission error or network failure. The collision recovery procedure is then triggered to ensure that the emergency message is delivered to the hot spot area. Note that this timeout period is different from the timeout of hot spot area discussed in next subsection C. The value of timeout (i.e., $T O_{1}$ ) is calculated as follows:

$$
T O_{1}=T_{\text {slot }} \times \frac{\text { Area }}{O_{x} O_{y}}
$$

where Area is the size of the transmission area. The value of Area/ $\left(O_{x} * O_{y}\right)$ is the total number of blocks in the transmission range. It is also the maximum number of deferrals during the transmission because we assign one deferral time slot to each block, as shown in Fig. 5. Therefore, Equation (2) means that if the timeout period expires, no vehicle can forward the message in all sectors due to network failure and no vehicle can receive the message successfully. Since vehicles cannot distinguish between collisions, transmission errors and network failure, we assume all forwarding failures are caused by collisions when re-forwarding the message. If there is more than one vehicle in a block, they will have the same deferral time, so a transmission collision will occur. Therefore, the transmission area should be divided into smaller block units when a collision happens. In our recovery process, the source vehicle rebroadcasts the failed message with the block flag set to 1. Afterwards, the receiving vehicle knows that it should use the smaller block size to re-calculate its deferral time. The collision problem will be resolved when the minimum block size is used.

1) The Value of the Block Size: the Case of Regular Segmentation: The initial block size of a relay is an important factor in the performance of our protocol because there is a tradeoff between the block size, which yields different collision probabilities, and the average deferral time. Larger blocks reduce the average deferral time of vehicles since there are fewer blocks, but it leads to higher collision probabilities. Therefore, it is very difficult to determine the optimal block size for different vehicle densities. In our mechanism, we find the optimal block size as follows. Interestingly, we do not need to consider the average deferral times of vehicles in the transmission area. The most important issue is the probability that the vehicles in several of the farthest blocks can relay the message successfully because all the vehicles have short deferral times. We found that the probability is high if we choose an appropriate block size for a certain vehicle density. We formulate the problem as follows.

Given an area $A$, we divide it into $m * n$ blocks whose size is $x^{*} y$. We define the $K$ border area as the $K$ farthest vertical areas from the source, as shown in Fig. 6. In practice, we let $15 \mathrm{~m}^{*} 4 \mathrm{~m}$ be the unit block size because it is close to the size of a car (i.e., $15 \mathrm{~m}$ includes the safe distance between two 


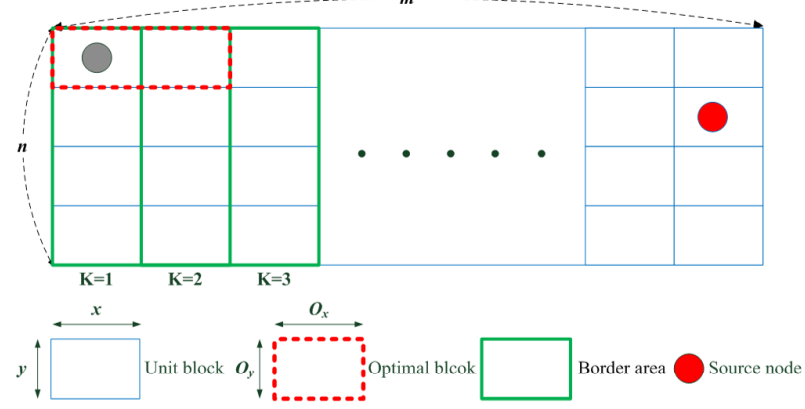

Fig. 6. Illustration of the K-border area.

cars). Thus, there is at most one vehicle in each unit block. Consequently, we can estimate the probability (i.e., $P_{K}$ ) that there is at least one vehicle in the $K$ border area that can forward the message successfully. Equation (3) calculates the probability of successful forwarding under the unit block size. A collision cannot occur in this situation because there only has one car in each block.

$$
P_{K}=\left\{\begin{array}{c}
\sum_{i=1}^{K \cdot n}\left(\begin{array}{c}
N_{A}-i \\
N_{c}-1
\end{array}\right) /\left(\begin{array}{c}
N_{A} \\
N_{c}
\end{array}\right), 0<N_{c} \leq N_{A}-K \cdot n \\
1, N_{A}-K \cdot n<N_{c} \leq N_{A}
\end{array}\right.
$$

where $K$ is the number of border areas, $N_{c}$ is the number of cars in area $A$, and $N_{A}$ is the total number of blocks in area $A$. We adjust the block size if it does not yield an optimal result. After adjusting the block size, the probability of success (i.e., $P_{K}^{\prime}$ ) is calculated as follows:

$$
P_{K}^{\prime}=\left\{\begin{array}{c}
\sum_{i=1}^{K \cdot N_{b c}}\left(\begin{array}{c}
x^{\prime} y^{\prime} \\
1
\end{array}\right)\left(\begin{array}{c}
N_{A}-x^{\prime} y^{\prime} i \\
N_{c}-1
\end{array}\right) /\left(\begin{array}{c}
N_{A} \\
N_{c}
\end{array}\right), 0<N_{c} \leq N_{A}-x^{\prime} y^{\prime} \\
0, N_{c}>N_{A}-x^{\prime} y^{\prime}
\end{array}\right.
$$

where $x^{\prime}$ is the length of the new block, $y^{\prime}$ is the width of the new block, and $N_{b c}$ is the number of blocks in the border area (i.e., $\left\lceil\frac{n}{y^{\prime}}\right\rceil$ ). By adjusting the block size, we can obtain the different value of $N_{b c}$.

The estimation results of aggregating the probabilities of $\mathrm{K}=1,2$ and 3 are shown in Fig. 7. From Figs. 7(a) to 7(c), we observe that: (1) a larger block size should be chosen when the vehicle density is low, and smaller block size should be used when the density is high, which is the same as our intuition; (2) for all vehicle densities, the probability of successful forwarding in a border area are high even if $K=1$, so long as we choose a suitable block size; and (3), in the high vehicle density scenario, we only need to use the unit block size without considering the large average deferral time because there is a very high probability that a vehicle with a very small deferral time (i.e., in the K-border area) will be able to forward a message successfully. Note that, although the probability of forwarding a message successfully in the K-border area with 16 blocks is lower than that of other block sizes, we can also use 16 blocks when the vehicle density is low (e.g., below 9). This is because the number of blocks in the transmission area is lower, which results in a low average deferral time. Therefore, we can adjust the block size dynamically based on the vehicle density. For example, if the vehicle density is around 5, the initial number of blocks should be 16, and it should be 4 blocks if the density is around 9. If the density is more than 13, we can just use the smallest block size.

2) The Value of the Block Size: the Case of Irregular Segmentation: We can obtain the probability distributions of the deferral times via Equation (4). Figures 8(a) to 8(e) show the cumulative probability distributions of the deferral times under different vehicle densities and block sizes. In Fig. 8(a), the vehicle selects the minimum block size (i.e., 1 unit) to segment the area. We observe that the vehicle has higher probability to have the lower deferral time when the car density is high. This situation means that it has higher probability to find out the car in the K-border area. According to the figures, the further blocks in the border area are assigned a shorter deferral time. In Fig. 8(e), the vehicle selects a huge block size (i.e., 16 units) to segment the area. Unfortunately, the probability is almost equal to zero under high car density environment because the transmission collision occurs frequently. On the contrary, the vehicle has higher probability to own the lower deferral time under huge block size segment method when the car density is low. The results demonstrate that different vehicle densities and block sizes affect the probability distribution of the deferral times. Hence, we should consider the vehicle density when adjusting the corresponding block size. Figures 9(a) to 9(d) show the successful delivery ratio under different car densities and segmentation methods. These two irregular segmentation methods generate two kinds of optimal block forms. In Fig. 6 , the value of y is fixed in horizontal segmentation method when the vehicle adjusts the optimal block size; the value of $\mathrm{x}$ is fixed in vertical segmentation method when the vehicle adjusts the optimal block size.

We observe that: (1) the horizontal segmentation method (e.g., $30 \mathrm{~m} * 4 \mathrm{~m}$ ) outperforms the vertical segmentation method (e.g., $15 \mathrm{~m} * 8 \mathrm{~m}$ ), so we suggest using the horizontal method to set the block size; and (2) a larger block size should be chosen when the vehicle density is low, and a smaller block size should be chosen when the density is high. According to the car density, the vehicle selects the most suitable block size, which has the highest probability of successful delivery, to segment the area and then calculate the deferral time of each block. Since we acquire the probability of successful delivery and the deferral time of each block under the $x^{*} y$ segmentation method, the expected deferral time is determined as follows:

$$
E_{x, y}=e_{x, y}+\sum_{i=1, j=1}^{x-1, y-1} e_{i, j}\left[1-P\left(\bigcup_{m=i+1, n=j+1}^{x, y} p_{m, n}\right)\right],
$$

where $E_{x, y}$ is the expected deferral time under the $x * y$ segmentation method, $e_{x, y}$ is the average deferral time under the $x * y$ segmentation method, and $p_{m, n}$ is the probability of successful delivery under the $m * n$ segmentation method. Based on the above equation, we can obtain the expected deferral times under different vehicle densities and block sizes. Moreover, we observe that the higher car density environment has higher probability to obtain the lower expected deferral times (e.g., the deferral time is about 1 2 time slot in high car density environment). As a result, the vehicle achieves the minimum deferral time if it chooses an optimal block size. 


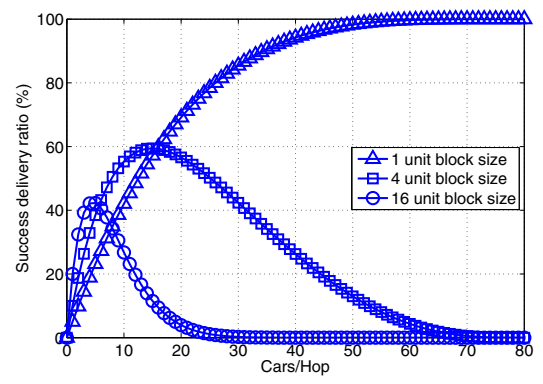

(a)

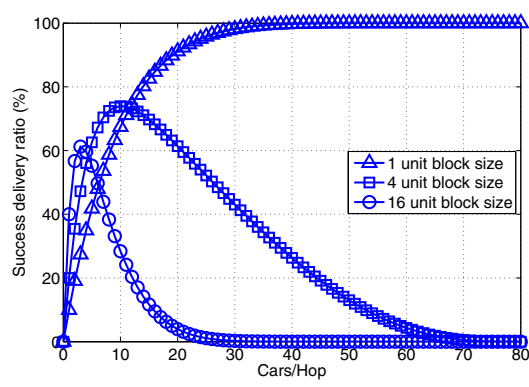

(b)

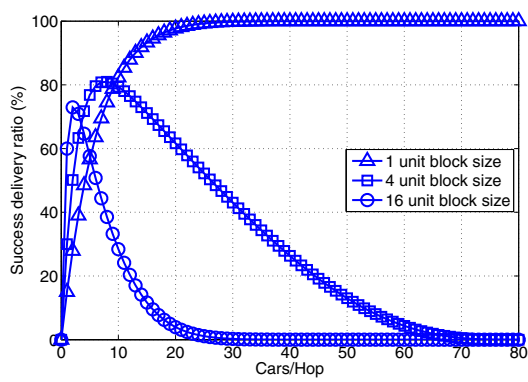

(c)

Fig. 7. Successful delivery ratio vs. car density in different sized blocks (a) $K=1$ (b) $K=1+2$ (c) $K=1+2+3$.

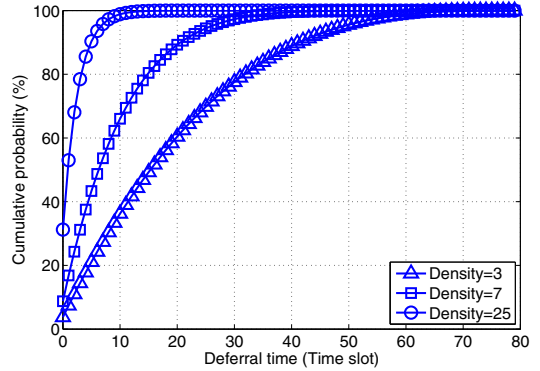

(a)

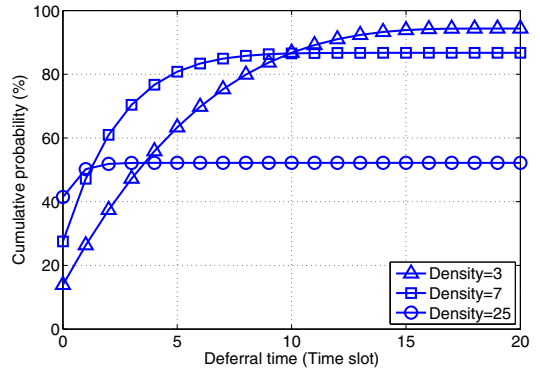

(c)

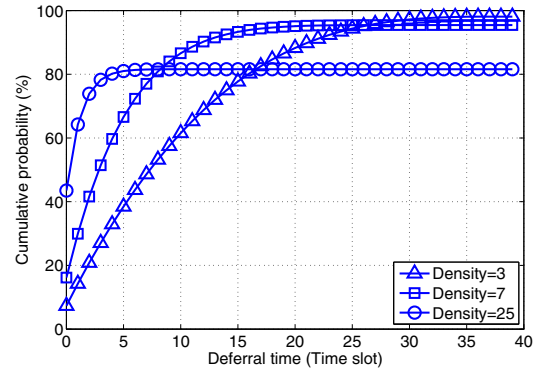

(b)

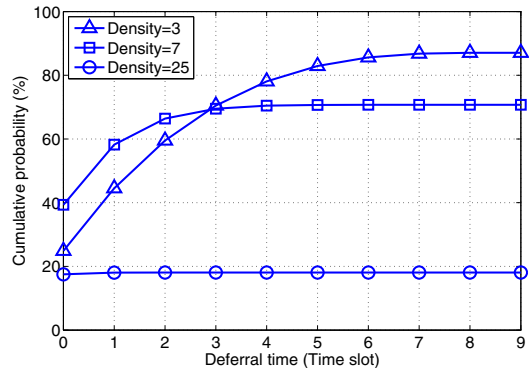

(d)

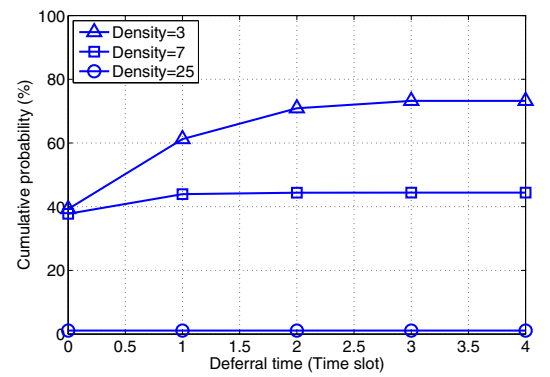

(e)

Fig. 8. The cumulative probability distribution of the deferral time under different car densities and block sizes (a) 1 unit block (b) 2 unit block (c) 4 unit block (d) 8 unit block (e) 16 unit block. 


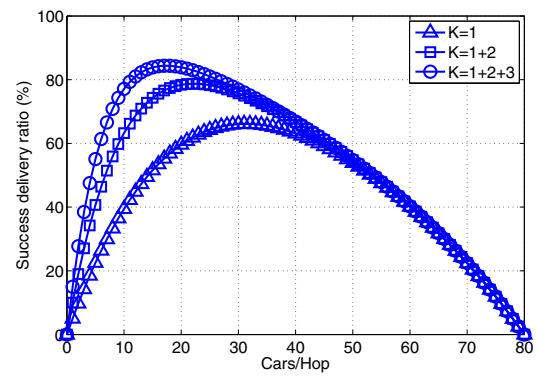

(a)

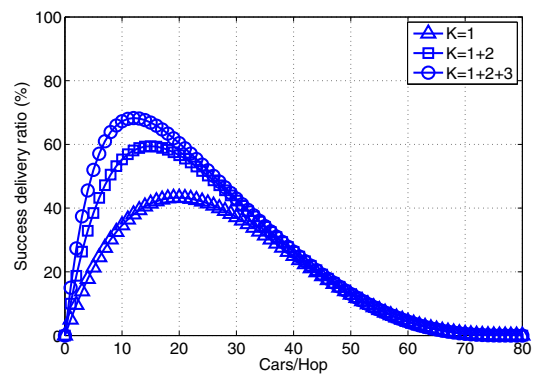

(c)

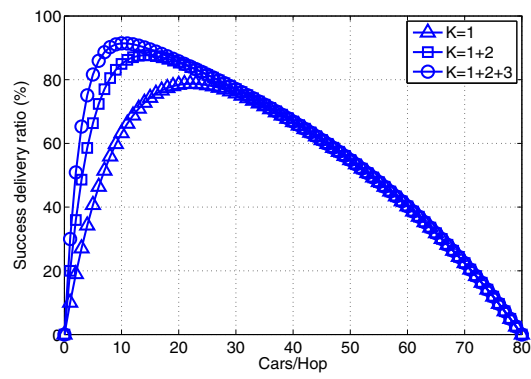

(b)

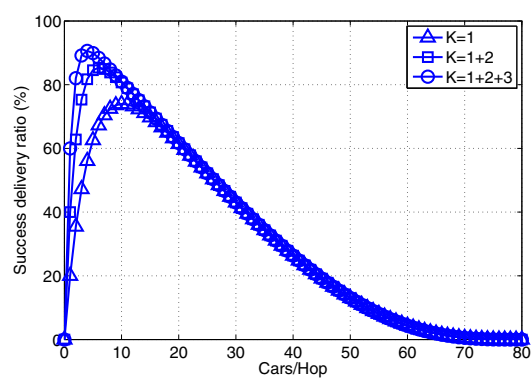

(d)

Fig. 9. Successful delivery ratio vs. car density under different segmentation methods (a) Block size is $15 \mathrm{~m} * 8 \mathrm{~m}$ ( 2 unit block size) (b) Block size is $30 \mathrm{~m} * 4 \mathrm{~m}$ ( 2 unit block size) (c) Block size is $15 \mathrm{~m} * 16 \mathrm{~m}$ (4 unit block size) (d) Block size is $60 \mathrm{~m} * 4 \mathrm{~m}$ (4 unit block size).

\section{Reliability Mechanism}

In the hot spot area (i.e., segments $\mathrm{A}$ and $\mathrm{C}$ in Fig.2), the message must be rebroadcasted repeatedly to satisfy the reliability criterion. Here, we explain how to compute the cycle (i.e., $\mathrm{TO}_{2}$ ) for rebroadcasting. Let $P_{\text {success }}$ be the reliability threshold (e.g., 99\%). Given the channel error ratio $P$, first we want to determine the transmission time $k$ that lets the probability of each vehicle in the transmission range receiving the message at least once be larger than $P_{\text {success }}$. We use the following equation:

$$
1-\sum_{i=1}^{n} C_{i}^{n} P^{i k} \geq P_{\text {success }},
$$

where $n$ is the number of vehicles in the transmission range. The summation in Equation (6) is the probability that $i$ cars will not receive the message in all $k$ rebroadcasts. We also calculate how long it takes a car to traverse the transmission range (i.e., $\left.T_{c a r}\right)$ as follows:

$$
T_{\text {car }}=\frac{L_{\text {range }} * 3.6}{\text { Speed }},
$$

where $L_{\text {range }}$ is the length of the transmission range per hop and Speed is the car speed in $\mathrm{km} / \mathrm{hr}$. Finally, we can calculate the period for rebroadcast by

$$
\mathrm{TO}_{2}=\frac{T_{c a r}}{k} .
$$

Thus, every vehicle around the exit is guaranteed to receive the message in time so that it can leave the freeway and avoid the traffic jam. If a vehicle moves out of the hot spot area (i.e., the vehicle moves from segment $\mathrm{C}$ to segment $\mathrm{B}$ in our scenario) and does not find out a relay vehicle, the network will become a disconnection network. To alleviate this adversary, our recovery mechanism is triggered (as described in the subsection B) that the vehicle in segment B still keeps rebroadcasting the message after a timeout period (i.e., $T O_{1}$ ).

\section{PERformance EVAluation}

In the performance analysis, we use the NS-2 simulator [23] [24] to implement our protocol. We also compare DEEP with the SB scheme [14], the Role-based Multicast (RBM) scheme [16], the Slotted 1-Persistence Broadcasting (S1PB) scheme [15] [18], and the Location-Based Scheme (LBS) [25]. The network topology is a $30 \mathrm{~km} * 16 \mathrm{~m}$ four-lane freeway scenario with a random node distribution and $100 \mathrm{~km} / \mathrm{hr}$ car speed. We assume that the average length of a vehicle is $4 \mathrm{~m}$. The node density in one transmission hop is classed as low (3 cars), medium ( 7 cars) and high (25 cars). The hot spot area is defined at the end of the linear road with a two-hop transmission range. The transmission range is set at $300 \mathrm{~m}$, and the data rate is set at $11 \mathrm{Mbps}$ as the default. The length of hot spot area is $500 \mathrm{~m}$ and $P_{\text {success }}$ is $99 \%$. Given the vehicle density (i.e., the number of vehicles in one transmission range), the vehicles are randomly distributed in the lanes. Each simulation result is the average of 100 runs. The performance metrics are the average propagation delay, the successful delivery ratio to the hot spot area, reliability in the hot spot area, and the saved broadcast ratio. (1) Average propagation delay: The delay is the most important criterion in an emergency/safety message application. The propagation delay means the length of time between when a message is transmitted by the AV and when it is received by the vehicles in the hot spot area. If a collision occurs, the node must rebroadcasted the message, which increases the delay. We measure the delay for different distances under other protocols. (2) Successful delivery ratio to the hot spot area: This metric 


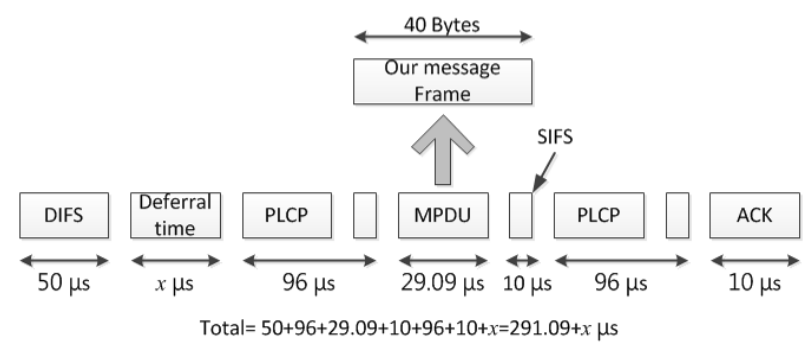

Fig. 10. The time period of one transmission.

\begin{tabular}{|l|c|c|c|c|c|c|c|c|}
\hline $\begin{array}{c}\text { Frame } \\
\text { Control }\end{array}$ & Message ID & $\begin{array}{c}\text { Source } \\
\text { Node ID }\end{array}$ & State & Flag & Source X & Source Y & Source Z & FCS \\
\hline 2 Bytes 4 Bytes 4 Bytes 1 Bytes 1 Bytes 8 Bytes 8 Bytes 8 Bytes 4 Bytes
\end{tabular}

Fig. 11. The message format.

means that the successful ratio of message transmitted from the source node to the hot spot area (i.e., segment $C$ ) during an event. In segment B, a message may not be transmitted to the segment $\mathrm{C}$ successfully due to a collision, channel error or network failure. If the forwarding mechanism does not deal with these problems, the message would be dropped or lost in the forwarding process. Thus, the vehicles in hot spot area would not receive the message, and miss the chance to leave the freeway. (3) Reliability in the hot spot area: $(u$ $v) / u$, where $u$ is the whole numbers of vehicles in the hot spot area and $v$ is the numbers of vehicles that they do not receive the emergency messages. A good recovery scheme can cope with the forwarding failure problem and improve the reliability. (4) Saved broadcast ratio: $(\beta-\alpha) / \beta$, where $\beta$ is the broadcast overhead via the flooding method and $\alpha$ is the broadcast overhead that vehicles actually broadcast the number of messages.

\section{A. Message Format}

Figure 10 shows the time period of one transmission and Fig. 11 shows the message format of the proposed protocol. We insert a message ID and source node ID to identify a message. The state field records the location state of the vehicle, and the flag field is turned on if the forwarding procedure fails. Based on the flags, vehicles reduce their optimal block size to avoid further collisions. Finally, the GPS location coordinate information is recorded.

\section{B. Average Propagation Delay}

From Fig. 12, we observe three phenomena about the average propagation delay and the block size. First, different block sizes achieve the minimum delay under different car densities. The car density is lower, the block size will be larger; conversely, the car density is higher, the block size will be smaller. Second, if we choose the appropriate block size, we obtain the smallest average delay. Third, in the high car density scenario, we choose the smallest block size, but we note that the average delay is minimal. The reason is that there is a high probability that a vehicle in the border area can forward the message successfully. Moreover, the simulation results match the above discussions of Equations (4) and (5). Using a small

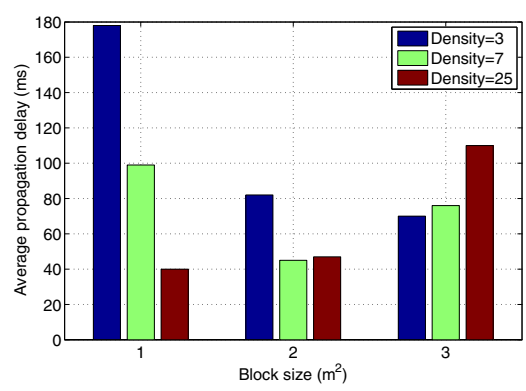

Fig. 12. Average delay vs. block size.

block size in a high density node area will yield a low average delay. Figures 13(a) to 13(c) show the average propagation delay for different distances between segment $\mathrm{A}$ and segment C. The larger the number of vehicles on the road, the higher will be the collision probability. DEEP obviously achieves the minimum delay in propagating emergency messages under different car densities because it ensures the farthest relay node having the least deferral time. The LBS protocol also achieves a low delay, but it does not guarantee that the emergency message is forwarded to the segment $\mathrm{C}$. Note that the successful delivery ratio is also very important in safety message dissemination.

\section{Successful Delivery Ratio to The Hot Spot Area}

A good message dissemination protocol should include a recovery scheme that can cope with the forwarding failure problem. Figures 14(a) and 14(b) show the successful delivery ratio, from the source to the hot spot area, for all the compared protocols under different distances channel error ratios in segment B. Generally, as the distance increases, the delivery ratio decreases due to the probability of collisions or network failure increases. However, DEEP still achieves a high delivery ratio, while RBM has the worst result because it lacks a good collision recovery mechanism. Figure 15 shows the delivery ratios for different car densities. Once again DEEP yields the best result. In sum, most schemes do not take the channel error and recovery mechanism into account. LBS scheme only rebroadcasts the message once in order to reduce the overhead but it cannot guarantee the delivery ratio. Oppositely, DEEP is based on repetition scheme to guarantee the high delivery ratio and reliability.

\section{Reliability in The Hot Spot Area}

A high successful delivery ratio implies high reliability. Figure 16 shows the successful delivery ratio of each protocol under different channel error ratios in the hot spot area (i.e., segment C). Most papers do not take the effect of the channel error into account. The RBM protocol yields the worst performance because it lacks a collision recovery mechanism. In contrast, the proposed protocol handles the collision problem well. Based on Equations (6) to (8), emergency messages are rebroadcasted repeatedly in a specific time period in hot spot area to satisfy the reliability criterion in DEEP for different vehicle densities and channel error ratios. Therefore, our protocol achieves the threshold for reliable transmission (i.e., 


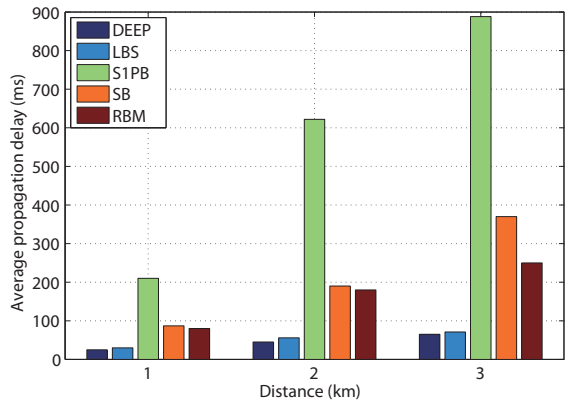

(a)

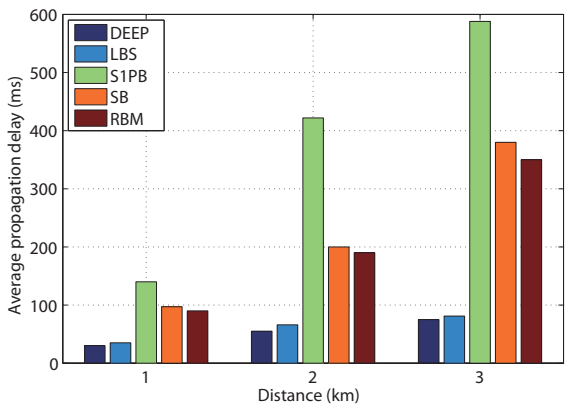

(b)

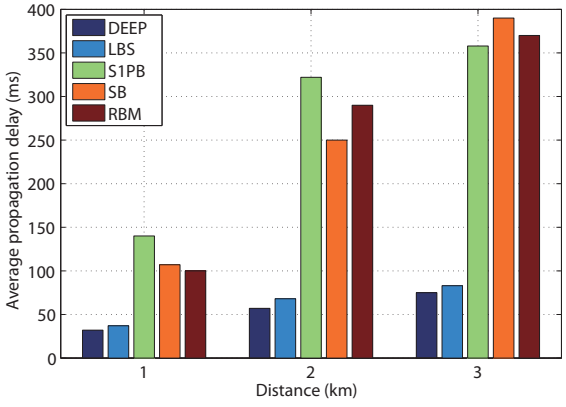

(c)

Fig. 13. Average delay based on the distance from the AV to the hot spot area (a) Low density (3cars/hop) (b) Medium density (7cars/hop) (c) High density (25cars/hop).

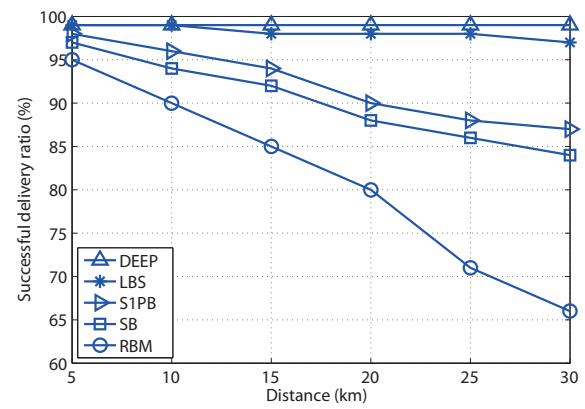

(a)

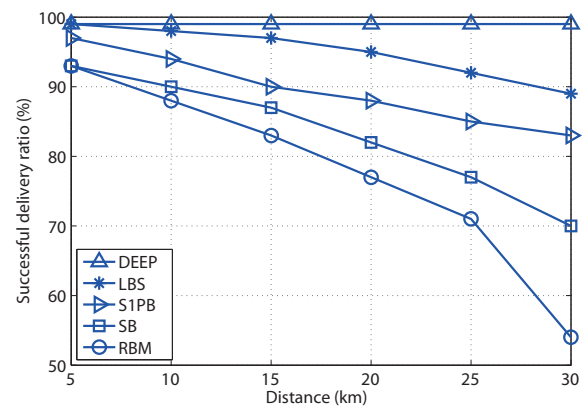

(b)

Fig. 14. Successful delivery ratio under different propagation distances with different channel error ratios (a) (Channel error ratio of segment $\mathrm{B}=1 \%)$ (b) (Channel error ratio of segment $\mathrm{B}=10 \%$ ).

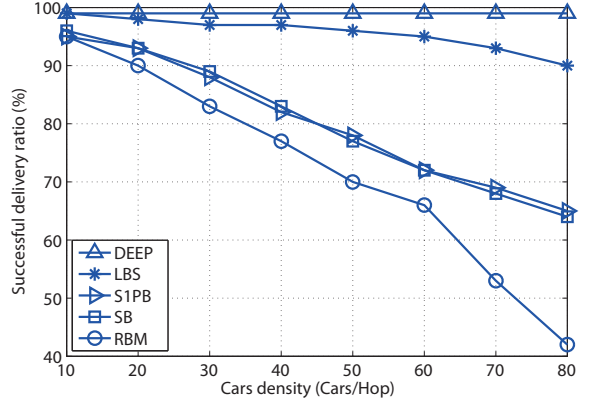

Fig. 15. Successful delivery ratio with different car densities.

the probability that all vehicles within the transmission range will receive the message at least once is more than $\left.P_{\text {success }}\right)$.

\section{E. Saved Broadcast Ratio}

Figure 17 shows the saved broadcast ratio under different car density. We can see that the existing schemes can save lots of broadcast overhead. Moreover, the performance is obviously improving when the car density is high. Our DEEP saves the fewer broadcast message because it would like to guarantee that the overall relevant vehicles receive the emergency message in segments A and C. In this paper, we focus on the dissemination of emergency messages where the successful delivery ratio and reliability are the most important indicators. In DEEP, the successful delivery ratio

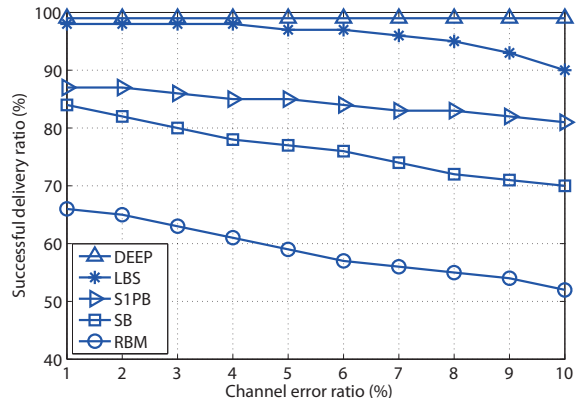

Fig. 16. Successful delivery ratio under different channel error ratios.

and reliability can be guaranteed under different car densities and channel error ratios (as shown in Fig. 14(b)). Although LBS saves the most message among compared schemes, the vehicle only rebroadcasts once which results in decreasing of the reliability and successful delivery ratio of messages to the hot spot area. Therefore, we think that the additional rebroadcast is worth especially for safety applications.

\section{CONCLUSiOnS AND Future WORK}

We have proposed a Density-aware Emergency message Extension Protocol (DEEP) in multi-lane freeway scenario. Under DEEP, a message is fast forwarded to the hot spot area and delivered to all vehicles in the area to satisfy the reliability criterion. Moreover, we find the appropriate initial 


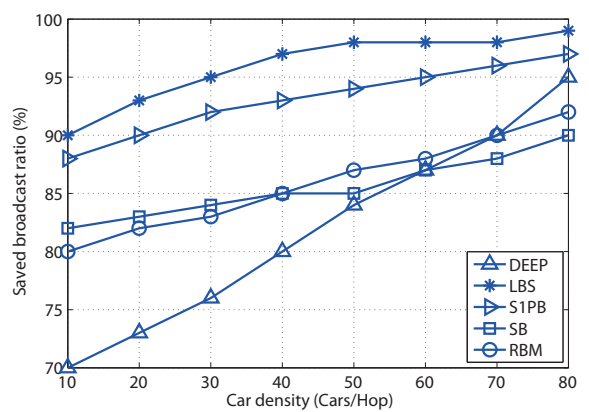

Fig. 17. Saved broadcast ratio under different car density.

block size for different car densities via a mathematical model. The simulation results demonstrate that the proposed protocol achieves low dissemination delay and high reliability while disseminating emergency messages. Because traffic information is distributed rapidly, drivers can change their routes and avoid traffic jams.

In the future, we will study two issues. (1) Heterogeneous message dissemination: the messages can be classified into safety and infotainment messages that they need to satisfy their QoS requirements, such as delay and reliability. Therefore, we will investigate the scenario with multiple heterogeneous source vehicles and propose a QoS dissemination mechanism to meet the requirements for mixed traffic. Moreover, ITS should base on car density, traffic conditions, and QoS requirements to adjust the dissemination mechanism to reduce the delay and overhead. (2) Complex environment: We are going to consider the complex environment such as urban VANETs. In fact, the road geometry (e.g., curve, crossover, etc.), the direction of vehicles, and buildings (i.e, obstacles) will affect the performance of the dissemination. Therefore, DEEP cannot be directly applied to some complex environment. We will incorporate the geocast and map-assisted techniques to achieve effective transmissions.

\section{REFERENCES}

[1] "Dedicated Short Range Communications (DSRC)." Available: http://grouper.ieee.org/groups/scc32/dsrc/index.html.

[2] T. Umedu, K. Isu, T. Higashino, and C. K. Toh, "An intervehicularcommunication protocol for distributed detection of dangerous vehicles," IEEE Trans. Veh. Technol., vol. 59, no. 2, pp. 627-637, Feb. 2010.

[3] X. Yang, J. Liu, and F. Zhao, "A vehicle-to-vehicle communication protocol for cooperative collision warning," IEEE MobiQuitous, pp. 114-123, Aug. 2004.

[4] A. Furda and L. Vlacic, "Enabling safe autonomous driving in realworld city traffic using multiple criteria decision making,.. IEEE Intell. Transp. Syst. Mag., vol. 3, no. 1, pp. 4-17, Spring 2011.

[5] P. Mohapatra, G. Chao, and J. Li, "Group communications in mobile ad hoc networks," IEEE Computer Mag., vol. 37, no. 2, pp. 52-59, Feb. 2004.

[6] Y.-C. Tseng, S.-Y. Ni, Y.-S. Chen, and J.-P. Sheu, "The broadcast storm problem in a mobile ad hoc network," ACM Wireless Networks, vol. 8, no. 2, pp. 153-167, Mar. 2002.

[7] Q. Xu, T. Mak, and R. Sengupta, "Vehicle-to-vehicle safety messaging in DSRC," ACM VANET, pp. 19-28, Oct. 2004.

[8] R. Chen, W.-L. Jin, and A. Regan, "Broadcasting in vehicular networks: issues and approaches," IEEE Netw. Mag., vol. 24, no. 1, pp. 20-25, Jan. 2010.

[9] I. Chisalita and N. Shahmehri, "A peer-to-peer approach to vehicular communication for the support of traffic safety applications," in Proc. 2002 IEEE International Conference on Intelligent Transportation Systems, pp. 336-341.
[10] P. Fan, "Improving broadcasting performance by clustering with stability for inter-vehicle communication," in Proc. 2007 IEEE Vehicular Technology Conference, pp. 2491-2495.

[11] M. Durresi, A. Durresi, and L. Barolli, "Emergency broadcast protocol for inter-vehicle communications," in Proc. 2005 IEEE International Conference on Parallel and Distributed Systems, pp. 402-406.

[12] Y.-C. Chu and N.-F. Huang, "An efficient traffic information forwarding solution for vehicle safety communications on highways," IEEE Trans. Intell. Transp. Syst., pp. 1-13.

[13] S. Yu and C. Cho, "Selective flooding method for propagating emergency messages in vehicle safety communications," in Proc. 2006 IEEE ICHIT, pp. 556-561.

[14] E. Fasolo, A. Zanella, and M. Zorzi, "An effective broadcast scheme for alert message propagation in vehicular ad hoc network," in Proc. 2006 IEEE ICC, pp. 3960-3965.

[15] N. Wisitpongphan, O. K. Tonguz, J. S. Parikh, P. Mudalige, F. Bai, and V. Sadekar, "Broadcast storm mitigation techniques in vehicular ad hoc networks," IEEE Wireless Commun. Mag., vol. 14, no. 6, pp. 84-94, Dec. 2007.

[16] L. Briesemeister and G. Hommel, "Role-based multicast in highly mobile but sparsely connected ad hoc networks," in Proc. 2000 ACM MobiHOC, pp. 45-50.

[17] J. Peng and L. Cheng, "A distributed MAC scheme for emergency message dissemination in vehicular ad hoc networks," IEEE Trans. Veh. Technol., vol. 56, no. 6, pp. 3300-3308, Nov. 2007.

[18] D. Sormani, G. Turconi, P. Costa, D. Frey, M. Migliavacca, and L. Mottola, "Towards lightweight information dissemination in intervehicular networks," 2006 ACM VANET, pp. 20-29.

[19] Y. Bi, L. X. Cai, X. (Sherman) Shen, and H. Zhao, "Efficient and reliable broadcast in intervehicle communication networks: a crossLayer approach," IEEE Trans. Veh. Technol., vol. 59, no. 5, pp. 2404 2417, June 2010.

[20] F. J. Ros, P. M. Ruiz, and I. Stojmenovic, "Acknowledgment-based broadcast protocol for reliable and efficient data dissemination in vehicular ad hoc networks," IEEE Trans. Mobile Comput., vol. 11, no. 1, pp. 33-46, Jan. 2012.

[21] Q. Xu, T. Mak, J. Ko, and R. Sengupta, "Medium access control protocol design for vehicle-vehicle safety messages," IEEE Trans. Veh. Technol., vol. 56, no. 2, pp. 499-518, Mar. 2007.

[22] Z. Wang and M. Hassan, "Blind XOR: low-overhead loss recovery for vehicular safety communications," IEEE Trans. Veh. Technol., vol. 61, no. 1, pp. 35-45, Jan. 2012.

[23] "Network simulator ns-2." Available: http://www.isi.edu/nsnam/ns.

[24] Q. Chen, D. Jiang, V. Taliwal, and L. Delgrossi, "IEEE 802.11 based vehicular communication simulation design for NS-2," 2006 ACM VANET, pp. 50-56.

[25] L.-W. Chen, Y.-H. Peng, and Y.-C. Tseng, "An infrastructure-less framework for preventing rear-end collisions by vehicular sensor networks," IEEE Commun. Lett., vol. 15, no. 3, pp. 358-360, Mar. 2011.

[26] Y. Gunter, B. Wiegel, and H. P. Grossmann, "Medium access concept for VANETs based on clustering," in Proc. 2007 IEEE Vehicular Technology Conference, pp. 2189-2193.

[27] P. Fan, P. Sistla, and P. Nelson, "Theoretical analysis of a directional stability-based clustering algorithm for VANETs," 2008 ACM International Workshop on Vehicular Ad Hoc Networks, pp. 80-81.

[28] H. Su and X. Zhang, "Clustering-based multichannel MAC protocols for QoS provisionings over vehicular ad hoc networks," IEEE Trans. Veh. Technol., vol. 56, no. 6, pp. 3309-3323, Nov. 2007.

[29] L. Bononi and M. Di Felice, "DBA-MAC: dynamic backbone-assisted medium access control protocol for efficient broadcast in VANETs," $J$. Interconnection Networks, vol. 10, no. 4, pp. 321-344, Oct. 2009.

[30] R. Aquino-Santos, V. Rangel-Licea, M. A. Garcia-Ruiz, A. GonzalezPotes, O. Alvarez-Cardenas, A. Edwards, M. G. Mayoral-Baldivia, and S. Sandoval-Carrillo, "Inter-vehicular communications using wireless ad hoc networks," a book chapter in Automotive Informatics and Communicative Systems: Principles in Vehicular Networks and Data Exchange. IGI Global, May 2009.

[31] K. M. Alzoubi, P. J. Wan, and O. Frieder, "New distributed algorithm for connected dominating set in wireless ad hoc networks," in Proc. 2002 IEEE Conf. on System Sciences, pp. 3849-3855. 


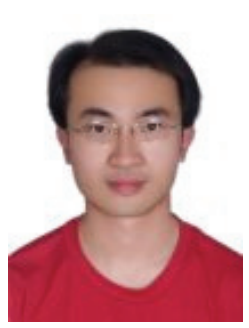

Ming-Chin Chuang received the Ph.D. degree in the Department of Computer Science and Information Engineering from National Chung Cheng University, Taiwan, in 2012. He is a Postdoctoral fellow in the Research Center for Information Technology Innovation, Academia Sinica, Taiwan. His research interests include mobility management, network security, cloud computing, and VANET.

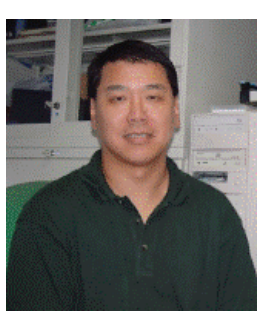

Meng Chang Chen received the Ph.D. degree in Computer Science from the University of California, Los Angeles, in 1989. He was with AT\&T Bell Labs from 1989 to 1992, New Jersey, USA and now he is a Research Fellow of Institute of Information Science, Academia Sinica, Taiwan. His current research interests include wireless network, QoS networking, information retrieval, data and knowledge engineering. 